\title{
Protocolo médico para la atención del infarto cerebral en el sistema de atención de llamadas de emergencia 9-1-1
}

\author{
Luis Daniel Sánchez-Arreola*
}

Subdirección Médica, Servicio de Urgencias, Hospital General La Villa, Secretaría de Salud, Ciudad de México, México; Capítulo de Prehospital de la Sociedad Mexicana de Medicina de Emergencia; Sistema de Atención de Emergencias 9-1-1, Centro Nacional de Información

\begin{abstract}
Resumen
En la actualidad, la enfermedad vascular cerebral (EVC) constituye una de las principales causas de mortalidad tanto en EE.UU. como en el continente europeo, caso similar en el resto de los países en vías de desarrollo. A pesar de los avances en el diagnóstico y tratamiento de este padecimiento, la EVC continúa siendo la sexta causa de muerte general y la primera causa de discapacidad en personas mayores de 50 años, además de ser también la segunda causa asociada a demencia. Lamentablemente hoy en día muchos pacientes continúan llegando tarde al hospital y fuera del periodo de ventana óptimo para ser sometidos a un tratamiento de reperfusión farmacológica. La activación de un servicio médico de urgencias prehospitalario y la solicitud de atención médica urgente constituyen el primer eslabón de la cadena de la sobrevida, la cual representa gráficamente los componentes y los momentos de atención del paciente que es víctima de una emergencia médica, cadena que va desde la fase prehospitalaria hasta el tratamiento definitivo en un hospital, lo cual denota la importancia del operador telefónico. La implementación del protocolo de infarto cerebral 9-1-1 sin duda será de gran utilidad para mejorar el diagnóstico temprano de los pacientes con datos clínicos de infarto cerebral, además de mejorar la gestión del traslado al hospital más cercano y la prenotificación, disminuyendo consecuentemente el tiempo puerta-aguja y permitiendo mejorar el porcentaje de pacientes sometidos a un tratamiento trombolítico efectivo con su consecuente tasa de éxito.
\end{abstract}

Palabras clave: Infarto cerebral. 911. Protocolo de emergencia. Ictus. Servicio médico de urgencias. Operador de emergencia.

\section{Stroke emergency protocol for the 9-1-1 emergency system}

\section{Abstract}

Currently, cerebrovascular disease (CVD) is one of the leading causes of death in the United States and the European continent, as similar as the rest of the developing countries. Despite advances in the diagnosis and treatment of this condition, cerebral vascular disease continues to be the sixth cause of general death and the leading cause of disability in people over 50 years of age, in addition to be the second cause associated with dementia. Unfortunately, today, many patients continue to arrive late to the hospital and outside the optimal window period to undergo pharmacological reperfusion treatment. The activation of a pre-hospital emergency medical service and the request for urgent medical attention is the first link in the chain of survival, which graphically represents the components and the moments of care of the patient who is victim of a

\section{Correspondencia:}

*Luis Daniel Sánchez-Arreola

Gregorio Gelati, 101

San Miguel Chapultepec

Del. Miguel Hidalgo

Fecha de recepción: 13-12-18

Fecha de aceptación: 03-01-19

DOI: 10.24875/REIE.M19000001
Disponible en internet: 08-02-2019

Rev Educ Investig Emer. 2019;1(1):3-7 www.medicinadeemergencias.com bajo la licencia CC BY-NC-ND (http://creativecommons.org/licenses/by-nc-nd/4.0/). 
medical emergency that starts from the prehospital phase until the definitive treatment in a hospital denoting the importance of the emergency dispatcher. The implementation of the 9-1-1 dispatcher stroke protocol will undoubtedly be very useful to improve the early diagnosis of patients with clinical data of stroke, in addition to improving the management of the transfer to the nearest hospital and the pre-notification decreasing consequently the door-needle time allowing to improve the percentage of patients subjected to effective thrombolytic treatment with its consequent success rate.

Key words: Cerebral infarction, 911. Emergency protocol. Stroke. Emergency medical services. Emergency dispatcher.

En la actualidad, la enfermedad vascular cerebral (EVC) constituye una de las principales causas de mortalidad tanto en EE.UU. como en el continente europeo, caso similar en el resto de los países en vías de desarrollo. A pesar de los avances en el diagnóstico y tratamiento de este padecimiento, la EVC continúa siendo la sexta causa de muerte general y la primera causa de discapacidad en personas mayores de 50 años, además de ser también la segunda causa asociada a demencia, después del Alzheimer ${ }^{1}$. Esto se debe mayormente a una falta de políticas públicas para mejorar la detección oportuna, el tratamiento adecuado y el manejo de sus complicaciones. En México la incidencia de EVC de tipo isquémico se ha incrementado en la población menor de 45 años debido a la presencia cada vez más frecuente de factores de riesgo como la obesidad, la diabetes mal tratada, la hipertensión arterial y el tabaquismo, lo que ocasiona una gran cantidad de muertes prematuras. ${ }^{2}$ En comparación con otros países miembros de la Organización para la Cooperación y el Desarrollo Económicos (OCDE), nuestro país reporta lamentables tasas de mortalidad, más elevadas a los 30 días del ingreso, por infarto cerebral isquémico en un hospital: 19.2 por cada 100 admisiones, cuando el promedio es de 8.2, lo que representa una tasa de incidencia de alrededor de 170 casos por cada 1,000 habitantes; la enfermedad vascular de tipo isquémico ha predominado en mayor proporción que el tipo hemorrágico ${ }^{3}$.

Se calcula que, en promedio, durante la fase aguda de un evento vascular cerebral, por cada minuto de isquemia cerebral no tratada o reperfundida se pierden aproximadamente 1.9 millones de neuronas, situación que es representada anatómicamente por la presencia de una zona de isquemia cerebral bien delimitada y destinada a ser permanente, rodeada de una zona periférica de mayor volumen caracterizada por un flujo sanguíneo cerebral deficiente y denominada «zona de penumbra». Esta zona se considera una región cerebral susceptible de ser recuperada en su totalidad si es posible realizar un tratamiento de reperfusión farmacológica o intervencionista en el menor tiempo posible, preferentemente antes de las $4.5 \mathrm{~h}$ como tiempo máximo de tolerancia. Para ello es importante considerar el lapso de tiempo que transcurre desde el inicio de los síntomas hasta que se recibe el tratamiento médico: cuanto menor sea el tiempo de inicio del tratamiento, mejores resultados se obtienen y mayor probabilidad de recuperación neurológica, casi o igual al 100\%. Este tiempo se considera el intervalo de isquemia ${ }^{3}$.

En referencia a este intervalo de isquemia, y siendo ampliamente conocido que el tiempo juega un factor importante en el éxito de la recuperación neurológica de los pacientes que cursan con un ictus, lamentablemente hoy en día muchos pacientes continúan llegando tarde al hospital y fuera del periodo de ventana óptimo para ser sometidos a un tratamiento de reperfusión farmacológica, tomando en cuenta que la mayoría de los pacientes acuden a las salas de urgencias por sus medios y menos del $20 \%$ lo hacen en ambulancia. Entre las causas que originan esta situación se encuentran el desconocimiento de los síntomas del ictus por parte de la población, la deficiencia en el reconocimiento de los síntomas y el retraso en el traslado por parte del personal prehospitalario, así como la falta de identificación y atención oportuna del paciente en el hospital ocasionada por la sobresaturación del Servicio de Urgencias, aspectos que desafortunadamente repercuten de manera directa en el pronóstico del paciente 4.

Dentro de la atención del paciente con ictus, la activación de un servicio médico de urgencias prehospitalario y la solicitud de atención médica urgente constituyen el primer eslabón de la cadena de la sobrevida, la cual representa gráficamente los componentes y los momentos de atención del paciente que es víctima de una emergencia médica, cadena que va desde la fase prehospitalaria hasta el tratamiento definitivo en un hospital $^{4}$. Por lo anterior el operador telefónico de emergencias posee un rol de suma importancia, ya que es él quien por medio de sus acciones facilita el punto de entrada y contacto del usuario con el sistema de salud y es la persona que puede facilitar la identificación de los datos clínicos de infarto cerebral mediante un interrogatorio sencillo, así como el auxilio o la atención inicial al paciente a través de un testigo o tercero, 


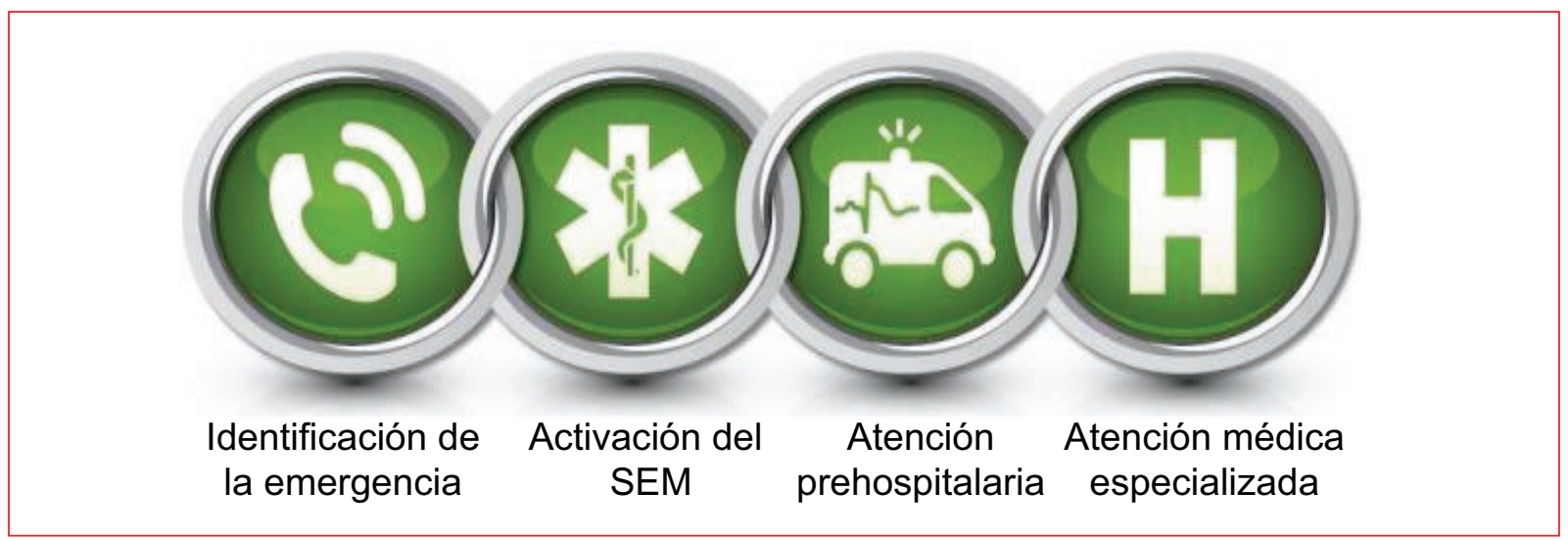

Figura 1. Cadena de la sobrevida de la atención prehospitalaria. Protocolo de Atención al Infarto Cerebral Para Operadores del 9-1-1. Sistema de Atención de Llamadas de Emergencia 9-1-1 (2017). SEM: Sistema de Emergencias Médicas

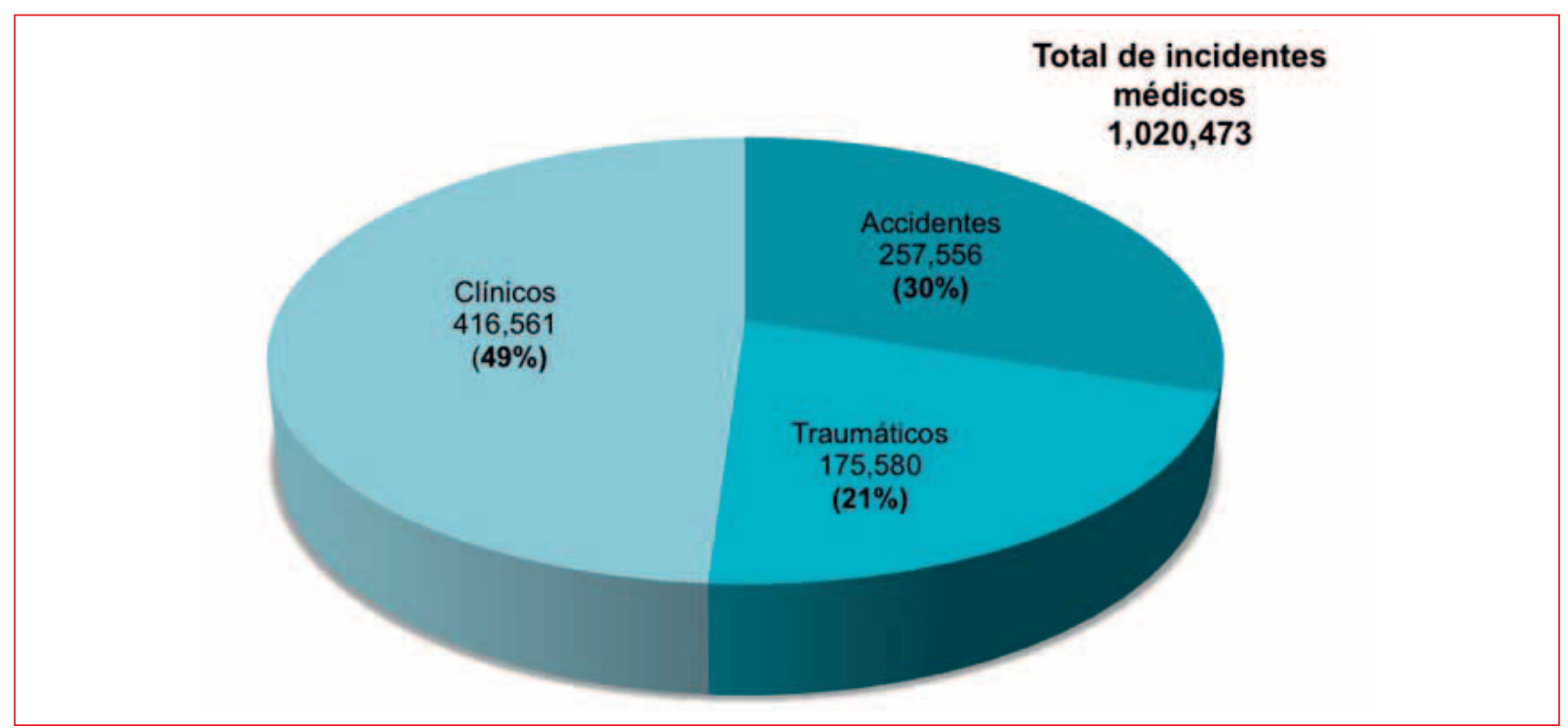

Figura 2. Participación relativa por subtipos de incidentes médicos en llamadas de emergencia procedentes en el periodo de enero a junio de 2018. Reporte estadístico de incidentes médicos, Centro Nacional de Información, SESNSP (2018).

canalizando los recursos necesarios para atender la emergencia en el menor tiempo posible y asegurando el traslado del paciente no al hospital más cercano sino al hospital que cuente con la capacidad resolutiva para brindar un tratamiento integral, situación que se conoce como la triple A (AAA): paciente adecuado, al hospital adecuado, en el tiempo adecuado (Fig. 1).

En México tan sólo en los meses de enero a junio de 2018 el servicio 9-1-1 a nivel nacional recibió aproximadamente 7,500,000 llamadas de emergencia, de las cuales cerca de 1,020,000 correspondieron a llamadas relacionadas con usuarios que reportaron emergencias médicas distribuidas en incidentes por padecimientos clínicos (49\%), accidentes $(30 \%)$ y lesiones traumáticas (21\%); se registró un total de 1,590 casos de incidentes relacionados con personas con datos clínicos de sospecha de infarto cerebral, lo que representa 8.8 casos diarios de probables ictus reportados a través del número único de emergencia 9-1-1 (Fig. 2).

Derivado de lo anterior, gracias a la suma de los esfuerzos de diferentes sociedades médicas, como la Sociedad Mexicana de Medicina de Emergencia, la Asociación Mexicana de Enfermedad Vascular 
Cerebral, la industria farmacéutica y el Servicio de Atención de Llamadas de Emergencia 9-1-1, fue posible la elaboración e incorporación al servicio 9-1-1 de un protocolo médico destinado a la atención del infarto cerebral, que se encuentra disponible hoy en los sistemas de cómputo del servicio 9-1-1 en 195 centros de atención de llamadas de emergencia distribuidos en todo el territorio nacional y que está disponible ya para más de 3,000 operadores telefónicos desde los últimos seis meses. Dicho protocolo médico fue diseñado con la finalidad de ayudar al operador telefónico a detectar los signos y síntomas de un infarto cerebral mediante preguntas cortas y sencillas, además de auxiliar a la persona que llama 0 al testigo en la escena mediante recomendaciones puntuales que le permitan brindar las primeras atenciones al paciente mientras arriba una ambulancia.

Para la detección de los datos clínicos de infarto cerebral, de forma posterior a la toma de datos primarios de contacto y localización (nombre, dirección, teléfono, etc.), el operador realiza una serie de preguntas fundamentadas en el acrónimo CAMALEÓN, creado y utilizado por primera vez en Costa Rica (https://www. youtube.com/watch?v=2cX6lpA709s), aprobado por la World Stroke Organization y la World Stroke Academy, y basado en los puntos de evaluación clínica de la escala prehospitalaria de Cincinnati. ${ }^{5}$ Se ha utilizado ya con gran éxito en una gran cantidad de países de Latinoamérica demostrando su utilidad para la detección temprana de signos clínicos de ictus. El acrónimo CAMALEÓN hace referencia a CA (debilidad en la mitad de la cara), MA (disminución de la fuerza en el brazo), LE (alteraciones del lenguaje) y ON (activación del teléfono y marcar 9-1-1 para reportar la emergencia), y sustituye a la tradicional escala de Cincinnati ya comentada y diseñada para la detección del ictus preferentemente por personal prehospitalario entrenado. Por su sencilla aplicabilidad demuestra un porcentaje de probabilidad para la detección del ictus del $42 \%$ ante la presencia de uno de los tres síntomas, del 64\% ante la presencia de dos y mayor al $97 \%$ ante la presencia de tres síntomas ${ }^{6}$.

Con la implementación de este nuevo protocolo médico de atención al infarto cerebral será posible plantear la meta de mejorar a mediano plazo la detección temprana del paciente con ictus, disminuir los tiempos de traslado al hospital y reducir el tiempo global de gestión de la emergencia a menos de $60 \mathrm{~min}$, fomentando que el personal prehospitalario realice una prenotificación al hospital, reduciendo sobre todo el tiempo puerta-aguja e incrementando el porcentaje de pacientes trombolizados y con el menor índice de secuelas posibles, pero sobre todo facilitando el reconocimiento de los signos de infarto cerebral desde el momento de la llamada de auxilio, logrando establecer un sistema de screening para dar prioridad a los casos con alta sospecha de un ictus y poder activar los protocolos de ayuda médica ${ }^{4}$.

Como parte complementaria a la incorporación de este nuevo protocolo médico, en el Servicio de Atención de Llamadas de Emergencia 9-1-1 se diseñaron e implementaron diversos esquemas de capacitación dirigidos a la población en general, al personal operativo de los centros de emergencia y al personal prehospitalario en los cuales se denota la importancia de la identificación de los síntomas de infarto cerebral y las indicaciones para realizar la activación del código Stroke o "código cerebro", protocolo diseñado para mejorar la atención de los pacientes con infarto cerebral con base en una secuencia de pasos establecidos; se busca aprovechar los recursos disponibles, prenotificar la llegada del paciente y organizar el proceso de atención hospitalaria para realizar el diagnóstico oportuno y administrar el tratamiento trombolítico lo antes posible. Este esquema de capacitación se imparte actualmente de manera periódica a más de 3,000 operadores telefónicos del 9-1-1 de todas las entidades federativas y su finalidad es la de brindar herramientas que faciliten la conformación de un sistema de atención homologado con énfasis en esta enfermedad.

La implementación del protocolo de infarto cerebral 9-1-1 sin duda será de gran utilidad para mejorar el diagnóstico temprano de los pacientes con datos clínicos de infarto cerebral, además de mejorar la gestión del traslado al hospital más cercano y la prenotificación, disminuyendo consecuentemente el tiempo puerta-aguja, lo que permitirá mejorar el porcentaje de pacientes sometidos a un tratamiento trombolítico efectivo con su consecuente tasa de éxito.

Es necesario continuar realizando acciones que permitan mejorar la detección y el tratamiento oportuno del paciente con infarto cerebral, así como diseñar un esquema de capacitación específico para el personal prehospitalario, acciones que sin duda requieren de la voluntad de todos los actores involucrados en la atención de estos pacientes: industria, gobierno, profesionales de la salud y comunidad. No olvidemos que por cada minuto que pasa la detección y el tratamiento efectivos pueden suponer la diferencia entre la vida 0 la muerte teniendo la oportunidad de ofrecer al paciente una vida libre de secuelas. 


\section{Bibliografía}

1. Cantú-Brito C, Ruiz-Sandoval JL, Chiquete E, Arauz A, et al. Factores de riesgo, causas y pronóstico de los tipos de enfermedad vascular cerebral en México: Estudio RENAMEVASC. Rev Mex Neurociencias. $2011 ; 12$.

2. OECD. Health at a Glance 2017: OECD Indicators. París: OECD Publishing; 2017.

3. Powers WJ, Rabinstein AA, Ackerson T, Adeoye OM, Bambakidis NC, Becker K, et al. 2018 Guidelines for the Early Management of Patients With Acute Ischemic Stroke: A Guideline for Healthcare Professionals
From the American Heart Association/American Stroke Association. Stroke. 2018;49(3):e46-e110.

4. Estadística Nacional del Número de Atención de Llamadas de Emergencia 9-1-1. Secretaría de Gobernación, Centro Nacional de Información, SESNSP, 2018. [Internet]. https://www.gob.mx/911/documentos/estadistica-nacional-del-numero-de-atencion-de-llamadas-de-emergencia-9-1-1-111029?state=published.

5. CAMALEON: una estrategia que salva vidas. [Internet]. http://contexto. cr/costa-rica/salud/2016/11/09/camaleon-una-estrategia-salvara-vidas/.

6. Meretoja A, Strbian D, Mustanoja S, Tatlisumak T, Lindsberg PJ Kaste M. Reducing in-hospital delay to 20 minutes in stroke thrombolysis. Neurology. 2012;79(4):306-13. 